\title{
Prevalence of Vitamin D Deficiency and Associated Risk Factors in the US Population (2011-2012)
}

\author{
Naveen R. Parva ${ }^{1}$, Satish Tadepalli ${ }^{2}$, Pratiksha Singh ${ }^{2}$, Andrew Qian ${ }^{1}$, Rajat Joshi ${ }^{3}$, Hyndavi Kandala ${ }^{1}$, \\ Vinod K. Nookala ${ }^{1}$, Pramil Cheriyath ${ }^{2}$ \\ 1. Internal Medicine, PinnacleHealth 2. Internal Medicine, Hackensack Meridian - Ocean Medical Center, Edison, USA \\ 3. Internal Medicine, Penn State Milton S. Hershey Medical Center
}

Corresponding author: Naveen R. Parva, naveenreddy.parva@gmail.com

\section{Abstract}

\section{Introduction}

1,25-dihydroxyvitamin D3 (cholecalciferol), the hormonally active form of vitamin D3, is a lipid-soluble compound that plays a significant role in clinical medicine due to its potent effects on calcium homeostasis and bone metabolism. Since foods containing natural vitamin D are rare, the primary source of the compound remains its nonenzymatic dermal synthesis through exposure to ultraviolet rays in sunlight. Although uncommon in most developed countries, recent literature has demonstrated that subclinical vitamin D deficiency can exist in certain populations and plays a role in downstream clinical consequences, including cardiovascular disease, cancer, diabetes, osteoporosis, and fractures. This study aims to identify the prevalence and change in the pattern of vitamin D deficiency in subpopulations throughout the United States to provide a foundation for further clinical studies correlating the clinical outcomes to vitamin deficiency.

\section{Methods}

Data analyzed in this study were collected through National Health and Nutrition Examination Survey (NHANES), specifically from a population of 4962 participants, age $\geqslant 20$ years, who were hospitalized between 2011 and 2012. This cohort was stratified to divide the population into patients that were vitamin D sufficient $(>50 \mathrm{nmol} / \mathrm{L}$ ) versus patients who were vitamin D deficient $(50 \mathrm{nmol} / \mathrm{L})$. The risk factors were compared between the subpopulations in 2005-2006 and 2011-2012.

\section{Conclusions}

The prevalence of vitamin D deficiency is greater in certain clinical subpopulations, and the presence of associated characteristics should raise the index of suspicion for the practicing clinician with regard to conditions associated with vitamin D deficiency, such as osteoporosis and osteomalacia. Further research investigating the pathophysiology of hypovitaminosis D and its clinical consequences can help better understand and prevent the development of associated comorbidities.

Categories: Internal Medicine, Preventive Medicine, Environmental Health Keywords: cholecalciferol, vitamin d deficiency, ergocalciferol, nhanes, cdc

\section{Introduction}

Vitamin D, a lipid-soluble vitamin, plays an essential role in maintaining skeletal integrity and function, electrolyte reabsorption, and immune system regulation among other health benefits. Vitamin D exists in two primary variants, vitamin D2 and D3. The hormonally active form of vitamin D, 1,25-dihydroxyvitamin D3 (cholecalciferol), plays a significant role in clinical medicine mainly due to its potent effects on calcium homeostasis and bone metabolism.

The primary source of vitamin D3 is sunlight, which contains ultraviolet rays that synthesize cholecalciferol in the skin [1]. Alternative sources of vitamin D3 include animal products, such as fatty fish, while vitamin D2 (ergocalciferol) can be obtained mainly from dietary plant and fungal sources such as mushrooms. Consequently, major risk factors for vitamin D deficiency include inadequate sunlight exposure, inadequate dietary intake of vitamin D-containing foods, and malabsorption syndromes such as Crohn's disease and celiac disease [2]. Although clinically apparent manifestations of vitamin D deficiency, such as rickets in children or periosteal bone pain in adults, are uncommon in most developed countries, recent literature suggests that subclinical, asymptomatic vitamin D deficiency still plays a notable role in contributing to several of the leading causes of death, including cardiovascular disease, cancer, and diabetes. 
Survey (NHANES), insufficient vitamin D levels were found in $41.6 \%$ of the 4495 -individual sample size. Race was identified as a significant risk factor, with African-American adults having the highest prevalence rate of vitamin D deficiency (82.1\%, 95\% CI, 76.5\%-86.5\%) followed by Hispanic adults (62.9\%; 95\% CI, 53.2\%-71.7\%) [3]. Additional risk factors for vitamin D deficiency that were identified included obesity, lack of college education, and lack of daily milk consumption [3].

This study aims to employ the most recently published NHANES data collected in 2011-2012 to identify the prevalence of hypovitaminosis D in subpopulations throughout the United States. Additionally, another objective of this study is to analyze trends in how rates of vitamin D deficiency are changing in these subpopulations with identified risk factors from 2005-2006 to 2011-2012. Multiple subpopulations previously unassociated with the risk of hypovitaminosis D were identified using this newly released data, including participants less than 65 years of age and participants who were cancer-free.

\section{Materials And Methods}

Data of participants were collected from NHANES, a program designed to study and assess the health and nutritional status of adults and children in the United States. The data from this site is released biannually and publicly available to researchers after data cleaning, editing, documentation, and Disclosure Review Board (DRB) review. As the data is available to researchers and is free of identifiers, no Institutional Review Board (IRB) approval and informed consent were required for this study.

Data analyzed in this study were collected through NHANES, specifically from a population of 4962 participants, 20 years of age and over. NHANES is a cross-sectional survey designed to monitor the health and nutritional status of the civilian non-institutionalized U.S. population. Data are collected and analyzed from a group of approximately 5000 individuals through the administration of standardized interviews and physical examinations that include laboratory tests utilizing blood and urine specimens. In 2011-2012, NHANES used a complex, multistage probability design to sample a civilian population from all 50 states, resolutely oversampling certain subgroups (non-Hispanic Asians, Hispanics, non-Hispanic AfricanAmericans, older adults, low-income whites/others) to increase the reliability and precision of data analysis for these subgroups. With these data, NHANES estimated trends in the prevalence, awareness, treatment, and control of select diseases in the U.S. population as a whole and in designated subgroups with selected diseases and risk factors.

Although there are differences in opinion regarding the optimal level of vitamin $\mathrm{D}$ in the body, most experts agree that levels less than $50 \mathrm{nmol} / \mathrm{L}$ are suboptimal for skeletal health [4]. Therefore, this value was selected to divide the population into patients who were vitamin $\mathrm{D}$ sufficient $(>50 \mathrm{nmol} / \mathrm{L})$ and vitamin $\mathrm{D}$ deficient $(<50 \mathrm{nmol} / \mathrm{L})$. The population was further stratified based on demographic information (age, gender, etc.), common health conditions (hypertension, hypercholesterolemia, rheumatoid arthritis, cancer, etc.), and lifestyle factors (milk consumption, exercise, etc.) to assess the relative prevalence of hypovitaminosis D in these subpopulations. The presence or absence of these variables in correlation with vitamin D levels was analyzed using logistic regression with a $95 \%$ confidence interval. P-values $<0.05$ were considered statistically significant.

A descriptive statistical analysis was performed using SAS version 9.4 for Windows (SAS Institute, Cary, NC, USA). The chi-square test, student t-test, and the Pearson correlation coefficient were used for the data analysis.

\section{Results}

The data of participants were collected from NHANES, a program designed to study and assess the health and nutritional status of adults and children in the United States. The data from this site are released biannually and publicly available to researchers after data cleaning, editing, documentation, and Disclosure Review Board (DRB) review. As the data is available to researchers and is free of identifiers, no IRB approval and informed consent were required for this study.

Of the 4962 participants interviewed in this study by NHANES 2011-2012, 1981 (39.92\%) were vitamin D deficient (serum levels less than $50 \mathrm{nmol} / \mathrm{L}$ ), a proportion that has remained consistent since 2005-2006, when NHANES found $40 \%$ of the population to be vitamin D deficient (Table 1 ). 


\section{Cureus}

\begin{tabular}{|c|c|c|c|c|c|c|}
\hline Vitamin D level & Number of People & Percent of People & Mean & Standard Deviation & Minimum & Maximum \\
\hline$<50 \mathrm{nmol} / \mathrm{L}$ & 1981 & $39.92 \%$ & 34.54 & 10.02 & 5.21 & 49.98 \\
\hline$\geq 50 \mathrm{nmol} / \mathrm{L}$ & 2981 & $60.08 \%$ & 76.73 & 22.69 & 50 & 373.67 \\
\hline Iotal Populatıon & 4962 & & & & & \\
\hline
\end{tabular}

\section{TABLE 1: Distribution of vitamin D ( Age $\geq 20$ )}

When comparing this vitamin D deficient population to the remaining 2981 participants with sufficient vitamin D levels (greater than or equal to $50 \mathrm{nmol} / \mathrm{L}$ ), several statistically significant correlations were found, each of which will be discussed below (Table 2).

\begin{tabular}{|c|c|c|c|c|}
\hline \multirow[b]{2}{*}{ Variables } & \multirow[b]{2}{*}{ Odds Ratio } & \multicolumn{2}{|c|}{ 95\% Confidence Interval } & \multirow[b]{2}{*}{$\mathrm{p}$-value } \\
\hline & & Lower Limit & Upper Limit & \\
\hline Age $\geq 65$ years & 0.52 & 0.44 & 0.61 & $<.0001$ \\
\hline Race: African-American & 3 & 2.61 & 3.44 & $<.0001$ \\
\hline Some college & 0.87 & 0.77 & 0.99 & 0.0351 \\
\hline Poor health & 1.44 & 1.23 & 1.69 & $<.0001$ \\
\hline Current smoker & 1.12 & 0.97 & 1.31 & 0.1308 \\
\hline Obesity (BMI $\geq 30$ ) & 1.31 & 1.15 & 1.49 & $<.0001$ \\
\hline Diabetes & 1.11 & 0.92 & 1.35 & 0.2837 \\
\hline Cancer & 0.69 & 0.54 & 0.89 & 0.0033 \\
\hline High total cholesterol (>200 mg/dl) & 0.83 & 0.72 & 0.96 & 0.01 \\
\hline Consume milk products daily & 0.63 & 0.55 & 0.71 & $<.0001$ \\
\hline
\end{tabular}

\section{TABLE 2: Risk factors for vitamin D deficiency}

\section{Race}

As previous studies indicate, significant differences in vitamin D levels were seen in non-Hispanic AfricanAmericans and Mexican-Americans as compared to other races. Non-Hispanic African-Americans composed a significant $39.3 \%$ of the vitamin D deficient population, compared to only $16.5 \%$ of the vitamin D sufficient population (p-value $<0.0001$ ). To a lesser extent, significant differences were also seen in MexicanAmericans (Table 3).

\begin{tabular}{|c|c|c|c|c|c|}
\hline \multirow{2}{*}{ Variables } & \multirow{2}{*}{\multicolumn{2}{|c|}{ Vitamin D Level <50 nmol/L }} & \multirow{2}{*}{\multicolumn{2}{|c|}{$\begin{array}{l}\text { Vitamin D Level } \\
\geq 50 \mathrm{nmol} / \mathrm{L}\end{array}$}} & \multirow{2}{*}{ p-value } \\
\hline & & & & & \\
\hline Total Population & \multicolumn{2}{|l|}{1981} & \multicolumn{2}{|l|}{2981} & \\
\hline Age $>65$ years & 294 & $14.84 \%$ & 774 & $25.96 \%$ & $<0.0001$ \\
\hline Gender (Female) & 1013 & $51.14 \%$ & 1494 & $50.12 \%$ & 0.4823 \\
\hline \multicolumn{6}{|l|}{ Race } \\
\hline Mexican-American & 237 & $11.96 \%$ & 258 & $8.65 \%$ & 0.0001 \\
\hline Other Hispanic & 196 & $9.89 \%$ & 312 & $10.47 \%$ & 0.5149 \\
\hline Non-Hispanic White & 377 & $19.03 \%$ & 1497 & $50.22 \%$ & $<0.0001$ \\
\hline
\end{tabular}




\section{Cureus}

\begin{tabular}{|c|c|c|c|c|c|}
\hline Non-Hispanic African-American & 778 & $39.27 \%$ & 493 & $16.54 \%$ & $<0.0001$ \\
\hline Others & 393 & $19.84 \%$ & 439 & $14.73 \%$ & $<0.0001$ \\
\hline \multicolumn{6}{|l|}{ Education } \\
\hline Didn't finish high school & 478 & $24.13 \%$ & 664 & $22.27 \%$ & 0.1285 \\
\hline Finished high school & 443 & $22.36 \%$ & 594 & $19.93 \%$ & 0.0387 \\
\hline Any college education & 1058 & $53.41 \%$ & 1723 & $57.80 \%$ & 0.0023 \\
\hline \multicolumn{6}{|l|}{ Health status } \\
\hline Good/excellent & 1279 & $64.56 \%$ & 2186 & $73.33 \%$ & $<0.0001$ \\
\hline Poor/fair & 476 & $24.03 \%$ & 508 & $17.04 \%$ & $<0.0001$ \\
\hline \multicolumn{6}{|l|}{ Smoking status } \\
\hline Never & 14 & $0.71 \%$ & 40 & $1.34 \%$ & 0.0347 \\
\hline Former & 365 & $18.43 \%$ & 771 & $25.86 \%$ & $<0.0001$ \\
\hline Current & 445 & $22.46 \%$ & 536 & $17.98 \%$ & 0.0001 \\
\hline Body weight & & & & & $<0.0001$ \\
\hline Underweight & 55 & $2.78 \%$ & 70 & $2.35 \%$ & \\
\hline Normal & 502 & $25.34 \%$ & 987 & $33.11 \%$ & \\
\hline Overweight & 582 & $29.38 \%$ & 997 & $33.45 \%$ & \\
\hline Obese & 842 & $42.50 \%$ & 927 & $31.10 \%$ & \\
\hline Hypertension & 680 & $34.33 \%$ & 1085 & $36.40 \%$ & 0.1356 \\
\hline Diabetes & 280 & $14.13 \%$ & 348 & $11.67 \%$ & 0.0107 \\
\hline High total cholesterol (>200mg/dL) & 479 & $24.18 \%$ & 840 & $28.18 \%$ & 0.0027 \\
\hline Low HDL cholesterol (HDL <40 mg/dL) & 359 & $18.12 \%$ & 513 & $17.21 \%$ & 0.3525 \\
\hline Consume milk products daily & 564 & $28.47 \%$ & 1256 & $42.13 \%$ & $<0.0001$ \\
\hline Thyroid stimulating hormone within normal range ( 0.4 - 4 mu/L) & 603 & $30.44 \%$ & 908 & $30.46 \%$ & 0.9878 \\
\hline Gout & 69 & $3.48 \%$ & 141 & $4.73 \%$ & 0.0326 \\
\hline Cancer & 112 & $5.65 \%$ & 300 & $10.06 \%$ & $<0.0001$ \\
\hline Coronary heart disease & 58 & $2.93 \%$ & 114 & $3.82 \%$ & 0.0909 \\
\hline Congestive heart failure & 69 & $3.48 \%$ & 99 & $3.32 \%$ & 0.7572 \\
\hline Liver disease & 76 & $3.84 \%$ & 121 & $4.06 \%$ & 0.6941 \\
\hline Depression & 306 & $15.45 \%$ & 413 & $13.85 \%$ & 0.1186 \\
\hline Psoriasis & 58 & $2.93 \%$ & 87 & $2.92 \%$ & 0.9848 \\
\hline Minutes on walking or bicycling for travel on a typical day - mean & $63.8(78.4)$ & $10-720$ & $64.7(73.9)$ & $10-600$ & 0.8272 \\
\hline
\end{tabular}

\section{TABLE 3: Demographics and risk factors}

The established physiologic mechanism by which non-Hispanic African-Americans and Mexican-Americans are predisposed to vitamin D deficiency is the increased melanin levels found in the skin. The increased melanin absorbs and scatters ultraviolet rays from sunlight, which results in the less efficient conversion of 7-dehydrocholesterol to previtamin D3, a precursor of vitamin D. Therefore, dark-skinned individuals with higher melanin content will experience slower vitamin D synthesis in comparison to light-skinned individuals. To further support this physiologic mechanism, data from the study shows that non-Hispanic white participants accounted for only $19.03 \%$ of the vitamin D deficient population while making up $50.22 \%$ of the vitamin D sufficient population ( $p$-value $<0.0001$ ). This is significant because it serves as evidence 
that not only is high melanin content in the skin a risk factor for vitamin D deficiency, but lower melanin content acts as a protective factor.

\section{Age}

Although NHANES only gathered data from participants at least 20 years old, age was still found to be a statistically significant factor in this study.

A total of $14.8 \%$ of people aged more than 65 years of age were vitamin D deficient. This data surprisingly shows an increased incidence of vitamin D deficiency among participants aged under 65 years of age, thus refuting a commonly accepted notion that increasing age is directly proportional to the risk of being vitamin D deficient.

\section{Education}

Another statistically significant factor found in this study was education, specifically high school and college education. Out of the vitamin D sufficient population, $57.80 \%$ were college-educated compared to $53.41 \%$ of the vitamin D deficient population ( $\mathrm{p}$-value=0.0023). This finding supports the notion that college-educated consumers have better health awareness in general than those who never attended college, resulting in statistically significantly lower rates of vitamin D deficiency.

\section{Health status}

The health status of the participants in the study was divided into binary categories of either “good/excellent” or “poor/fair." Among those who were vitamin D deficient, 64.56\% had good or excellent health status compared to $73.33 \%$ of those who were vitamin D sufficient (p-value $<0.0001$ ). Similarly, $24.03 \%$ of vitamin D deficient participants had poor or fair health status compared to $17.04 \%$ of vitamin-D sufficient patients ( $p$-value $<0.0001$ ). This variable is more difficult to extrapolate due to the nebulous nature of how each patient is categorized under an overarching health status. Regardless, the data support a direct correlation between the participants' overall health status and their vitamin D levels.

\section{Smoking}

In this study, participants were divided into three different smoking categories: never smoked, former smoker, and current smoker. Rates of vitamin D deficiency were statistically significant among the "former smoker" and "current smoker" subpopulations but not the "never smoked" subpopulation. Among the vitamin D deficient population, $18.43 \%$ were former smokers compared to $25.86 \%$ of the vitamin D sufficient population, thus making vitamin D deficiency less prevalent among former smokers ( $\mathrm{p}$-value $<0.0001$ ). An opposite trend was noted with current smokers; among the vitamin D deficient population, $22.46 \%$ were current smokers, whereas among the vitamin D sufficient population, $17.98 \%$ were current smokers ( $\mathrm{p}$-value $<0.0001)$.

Recent studies have shown that the correlation between smoking and hypovitaminosis D may be related to the ability of sinus mucosa to activate circulating vitamin D levels. A recent study by Mulligan and colleagues examined the effects of cigarette smoke extract on primary human sinonasal epithelial cells, which showed a decreased expression for the gene that activates vitamin D3 in smokers [5]. This ability of cigarette smoke to impair vitamin D3 activation is a novel mechanism by which smoking induces proinflammatory and carcinogenic effects.

\section{Body weight}

The study showed statistically significant differences in vitamin D deficiency rates with respect to weight, with $42.5 \%$ of vitamin D deficient participants falling under the category of obese (BMI $\geqslant 30$ ) compared to $31.1 \%$ of vitamin D sufficient participants ( $\mathrm{p}$-value $<0.0001$ ). This relationship between obesity and vitamin $\mathrm{D}$ deficiency is a consistent association found in published literature, with body fat content being inversely correlated to serum 25-hydroxyvitamin D concentrations [6]. Other factors that may contribute to this relationship include decreased mobility among obese patients, thereby decreasing sun exposure, as well as increased rates of bariatric or gastric bypass procedures, which may result in malabsorption of fat-soluble vitamins such as vitamin D.

Interestingly, the trend is reversed among overweight (BMI between 25 and 29.9) patients in this study, with $29.38 \%$ of vitamin D deficient participants being overweight compared to $33.45 \%$ of vitamin D sufficient participants (p-value $<0.0001$ ). Patients who are overweight but not obese are less likely to be prone to reduced mobility, leading to increased sunlight exposure, and they are not eligible for bariatric procedures that may induce a malabsorptive state and, consequently, hypovitaminosis D. Another hypothesis for why overweight patients are, in fact, less likely to be vitamin D deficient is that their diet may consist of more vitamin D-containing animal products than their non-overweight counterparts.

\section{Daily milk product consumption}


Milk fortification with vitamin D began in the United States during the 1930s, largely as an effort to combat rickets, a major public health problem at the time [7]. Almost all milk available in the U.S. is now fortified with $100 \mathrm{IU} /$ cup of vitamin D, consequently, making rickets a rare disease in the United States. In this study, participants were interviewed to identify those who consumed milk products on a daily basis. Of the vitamin D deficient subpopulation, $28.47 \%$ consumed milk products daily, whereas $42.13 \%$ of vitamin sufficient participants consumed milk products daily (p-value $<0.0001$ ). These data support the efficacy of vitamin Dfortified milk products as a method of prophylaxis against hypovitaminosis D in conjunction with daily sun exposure.

\section{Cancer}

Vitamin D has long been established as a protective factor for cancer, with multiple studies showing an increased risk of cancer among vitamin D deficient patients, including colon, breast, ovarian, and prostate cancer [8]. In this study, however, there is a statistically significant $(\mathrm{p}<0.0001)$ higher rate of cancer found among the vitamin D sufficient population (10.06\%) compared to the vitamin D deficient population (5.65\%).

\section{Diabetes}

The relationship between vitamin $\mathrm{D}$ deficiency and diabetes has long been explored, with growing evidence suggesting vitamin $\mathrm{D}$ deficiency is a contributing factor to the development of both type 1 and type 2 diabetes. Identified mechanisms for this relationship include the presence of vitamin D receptors on the Bislet cells in the pancreas responsible for secreting insulin, as well as clinical improvement in diabetic patients who receive vitamin D treatment through increased insulin secretion [9]. Lastly, the role of vitamin $\mathrm{D}$ in calcium homeostasis, specifically its ability to reabsorb calcium in the small intestine and kidney, has been shown to indirectly regulate insulin secretion [9].

The data collected in this study support these findings, with $14.13 \%$ of the vitamin D deficient participants having diabetes compared to $11.67 \%$ of the vitamin D sufficient participants ( $p$-value 0.0107$)$. Other factors may contribute to this correlation between vitamin D deficiency and diabetes, including increased rates of obesity, kidney damage, and renal failure in diabetics, leading to impaired hydroxylation of 25hydroxyvitamin D and, eventually, secondary hyperparathyroidism [10].

\section{Non-significant results}

The study stratified vitamin D deficient and sufficient populations by several other factors that yielded nonsignificant results, including gender, hypertension, thyroid-stimulating hormone (TSH), gout, coronary heart disease, congestive heart failure, liver disease, depression, and psoriasis. Additionally, daily minutes spent on walking or bicycling was another variable that proved to be non-significant, with similar results in both vitamin D deficient and sufficient subpopulations (p-value 0.83 ).

\section{Discussion}

Growing scientific evidence has implicated vitamin D deficiency in a multitude of chronic conditions, including type I diabetes, rheumatoid arthritis, hypertension, cardiovascular disease, and several common deadly cancers, among others [11]. With the growing prevalence of vitamin D deficiency across the United States and its association with these leading causes of mortality, it has become more important than ever to delineate vitamin D's role in the pathogenesis of these diseases and use data to pinpoint established risk factors for vitamin D deficiency.

This study was conducted primarily to analyze the prevalence of vitamin D deficiency in the United States based on the latest published data collected by NHANES 2011-2012. Of the 4962 participants surveyed and examined, 1981 (39.92\%) were found to be vitamin D deficient, which was in concordance with prior data collected in 2005-2006 (also by NHANES). A cross-sectional study was then conducted to further stratify the population to find factors associated with the risk of vitamin D deficiency, of which several were identified.

Primarily, this study supported established relationships between hypovitaminosis D and race, education, health status, smoking status, body weight, consumption of milk products, and diabetes. More specifically, the data from NHANES shows that there are statistically significant relationships between vitamin D deficiency and the non-Hispanic African-American as well as Mexican-American races, which was expected due to the increased levels of skin melanin found in these races on average. Similarly, data from this study show that there is an increased likelihood of vitamin D deficiency found among participants with diabetes, obesity, poor/fair health status, no college education, and current smokers. Lastly, the study supports the notion that the daily consumption of milk products serves as an effective prophylaxis against vitamin D deficiency.

Perhaps more interestingly, some of the data analyzed in this study refuted widely accepted associations between hypovitaminosis D and old age, as well as cancer. Prior studies have shown that increasing age is commonly associated with an increased risk of vitamin D deficiency, attributing this relationship to two main factors: the decreased efficacy of the human body's synthesis of vitamin D through UVB-irradiation 
Surprisingly, findings from this study deviate from the established notions that age correlates with the risk of vitamin D deficiency since we discovered that more participants under the age of 65 years of age were vitamin D deficient. Various confounding factors could contribute to these findings, but the surprising prevalence of vitamin D deficiency among individuals younger than 65 years of age compared to those older than 65 years is too drastic to be ignored. One primary factor that may play a role in decreasing the rates of hypovitaminosis D in older adults is the use of vitamin D supplementation, which has increased over time in the U.S. adult population. In one study by Rooney et al., less than $0.3 \%$ of the population had a daily supplemental intake of 1000 IU or more of vitamin D in 1999-2000, compared to 18.2\% in 2013-2014 [12]. Today, over children and adolescents, on the other hand, the percentage of participants who took vitamin D supplements has remained relatively stable throughout the years [13].

Another proposed hypothesis for why vitamin D levels seem to be decreasing in the younger population is the exponential increase in technology such as big-screen television, computers, and gaming devices, making them inclined to stay indoors as compared to prior generations and, consequently, having less sunlight exposure. Additionally, because the average age of retirement in the United States is around 65 years, participants under this cutoff age may be spending most of their days working indoors during the time the sun is out. A 2001 study conducted by the National Human Activity Pattern Survey (NHAPS) to assess for exposure to environmental pollutants showed that over $90 \%$ of survey respondents spent time inside a residence from about $11 \mathrm{pm}$ to $5 \mathrm{am}$, with respondents in schools, public buildings, offices, and factories spending time inside a residence between 7 am and 5 pm [14].

Our study showed that there was a statistically significant higher rate of cancer among the vitamin D sufficient population compared to those who were vitamin D deficient, thus contradicting strong evidence that vitamin D reduces the incidence and death rates of breast, prostate, colon, and ovarian cancers [8]. One factor that may contribute to this seemingly contradictory relationship may be the administration of vitamin $\mathrm{D}$ and other dietary supplements to patients diagnosed with cancer by their healthcare providers, whereas the vitamin D deficient population may be at high risk for cancer or even unaware of existing cancers and, consequently, not treated yet. Additionally, studies have also shown evidence that like low vitamin D levels, high vitamin D levels ( $>80 \mathrm{nmol} / \mathrm{L}$ ) may also contribute to the increased rates of cancer through the development of vitamin D resistance [8].

\section{Conclusions}

Although more research needs to be conducted to pinpoint the effects of age, occupation, technology, and cancer on vitamin D deficiency rates, the findings from this study are significant, showing that vitamin D deficiency is becoming an epidemic across the United States, even among groups that were not previously labeled “at-risk." Healthcare professionals must be aware of the growing prevalence of vitamin D deficiency not only among patients with established risk factors, such as African-American or Mexican-American race, obesity, or diabetes but also among younger individuals and those who have no history of cancer.

\section{Additional Information \\ Disclosures}

Human subjects: Consent was obtained by all participants in this study. Animal subjects: All authors have confirmed that this study did not involve animal subjects or tissue. Conflicts of interest: In compliance with the ICMJE uniform disclosure form, all authors declare the following: Payment/services info: All authors have declared that no financial support was received from any organization for the submitted work. Financial relationships: All authors have declared that they have no financial relationships at present or within the previous three years with any organizations that might have an interest in the submitted work. Other relationships: All authors have declared that there are no other relationships or activities that could appear to have influenced the submitted work.

\section{References}

1. Nair R, Maseeh A: Vitamin D: the "sunshine" vitamin. J Pharmacol Pharmacother. 2012, 3:118-126.

2. Dedeoglu M, Garip Y, Bodur H: Osteomalacia in Crohn's disease. Arch Osteoporos. 2014, 9:177. 10.1007/s11657-014-0177-0

3. Forrest KY, Stuhldreher WL: Prevalence and correlates of vitamin D deficiency in US adults . Nutr Res. 2011, 31:48-54. 10.1016/j.nutres.2010.12.001

4. Vitamin D deficiency in adults: definition, clinical manifestations, and treatment . (2014). Accessed: March 30, 2018: https://www.uptodate.com/contents/vitamin-d-deficiency-in-adults-definition-clinicalmanifestations-and-treatment.

5. Mulligan JK, Nagel W, O'Connell BP, Wentzel J, Atkinson C, Schlosser RJ: Cigarette smoke exposure is associated with vitamin D3 deficiencies in patients with chronic rhinosinusitis. J Allergy Clin Immunol. 2014, 134:342-349. 10.1016/j.jaci.2014.01.039

6. Vanlint S: Vitamin D and obesity. Nutrients. 2013, 5:949-956. 10.3390/nu5030949

7. Calvo MS, Whiting SJ, Barton CN: Vitamin D fortification in the United States and Canada: current status and data needs. Am J Clin Nutr. 2004, 80:1710-1716. 10.1093/ajcn/80.6.1710S 


\section{Cureus}

8. Garland CF, Garland FC, Gorham ED, Lipkin M, Newmark H, Mohr SB, Holick MF: The role of vitamin D in cancer prevention. Am J Public Health. 2006, 96:252-261. 10.2105/AJPH.2004.045260

9. Martin T, Campbell RK: Vitamin D and diabetes. Diabetes Spectr. 2011, 24:113-118. 10.2337/diaspect.24.2.113

10. Al-Badr W, Martin KJ: Vitamin D and kidney disease . Clin J Am Soc Nephrol. 2008, 3:1555-1560. 10.2215/CJN.01150308

11. Holick MF: The vitamin D epidemic and its health consequences . J Nutr. 2005, 135:2739-2748. 10.1093/in/135.11.2739s

12. Rooney MR, Harnack L, Michos ED, Ogilvie RP, Sempos CT, Lutsey PL: Trends in use of high-dose vitamin D supplements exceeding 1000 or 4000 international units daily, 1999-2014. JAMA. 2017, 317:2448-2450. 10.1001/jama.2017.4392

13. Bailey RL, Dodd KW, Goldman JA, et al.: Estimation of total usual calcium and vitamin D intakes in the United States. J Nutr. 2010, 140:817-822. 10.3945/jn.109.118539

14. Klepeis NE, Nelson WC, Ott WR, et al.: The National Human Activity Pattern Survey (NHAPS): a resource for assessing exposure to environmental pollutants. J Expo Anal Environ Epidemiol. 2001, 11:231-252. 10.1038/sj.jea.7500165 